Instructions for authors, subscriptions and further details:

\title{
http://rise.hipatiapress.com
}

\section{De la Antropología de la Sexualidad a la Sociología de la Diversidad}

Jaime De la Calle Valverde ${ }^{1}$

1) Universidad Nacional de Educación a Distancia (UNED), Spain

Date of publication: October $25^{\text {th }}, 2016$

Edition period: October 2016-February 2017

To cite this article: De la Calle Valverde, J. (2016). De la Antropología de la Sexualidad a la Sociología de la Diversidad. International Journal of Sociology of Education, 5(3), 190-213. doi: 10.17583/rise.2016.1990

To link this article: http://dx.doi.org/10.17583/rise.2016.1990

PLEASE SCROLL DOWN FOR ARTICLE

The terms and conditions of use are related to the Open Journal System and to Creative Commons Attribution License (CC-BY) 


\section{From Anthropology of Sexuality to Sociology of Diversity}

Jaime de la Calle Valverde

$U N E D$

(Received: 2 March 2016; Accepted: 1 August 2016; Published: 25 October 2016)

\section{Abstract}

UNED (Universidad Nacional de Educación a Distancia) was the first Spanish university to introduce courses on sexuality from a social and cultural standpoint. The name and the aim of the course: Anthropology of Sexuality. It was inserted as an academic subject to accomplish the Social and Cultural Anthropology Bachelor's Degree. And its teaching was established at the School of Sociological and Political Sciences (Department of Sociology I). The academic year 2003-2004 evinced the start of this innovating experience. For eleven years this educational and intellectual reality was alive: it last until the university implemented a new curriculum, the so called "Plan Bolonia". The plan had diverse derivations. For instance, it opened negotiations, and with it disagreements, among school and departments to distribute the new academic subjects of the educational project. At the final analysis, the outcome of the process was that Anthropology of Sexuality was eliminated of the degree on Social and Cultural Anthropology: "the Degree". Instead of it, Sociology of Diversity was introduced as a subject on the new sociological degree.

Keywords: UNED, anthropology of sexuality, Plan Bolonia, sociology of diversity, event 


\section{De la Antropología de la Sexualidad a la Sociología de la Diversidad}

Jaime de la Calle Valverde UNED

(Recibido: 2 Marzo 2016; Aceptado: 1 Agosto 2016; Publicado: 25 Octubre 2016)

\section{Resumen}

La Universidad Nacional de Educación a Distancia (UNED) fue la primera universidad española en impartir entre sus enseñanzas regladas una "Antropología de la Sexualidad" desde una perspectiva social y cultural. La andadura comenzó en el curso académico 2003-2004; la asignatura formó parte de los planes de estudio de la nueva Licenciatura de Antropología Social y Cultural adscrita a la Facultad de Filosofía, si bien la asignatura se impartió desde el Departamento de Sociología I de la Facultad de Ciencias Políticas y Sociología. La experiencia ha durado 11 cursos académicos, hasta la implantación definitiva del Plan Bolonia. El Plan Bolonia tuvo efectos diversos; por ejemplo, abrió las negociaciones y los desacuerdos entre facultades y departamentos para repartirse/distribuirse la docencia en diferentes asignaturas. El resultado, en el caso que presentamos, fue la desaparición de la Antropología de la Sexualidad del nuevo Grado en Antropología Social y Cultural y su conversión en una Sociología de la Diversidad en el nuevo Grado en Sociología.

Palabras clave: UNED, antropología de la sexualidad, Plan Bolonia, sociología de la diversidad, acontecimiento. 


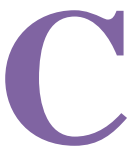

on el incremento del interés de las ciencias sociales por la sexualidad, especialmente en las dos últimas décadas del siglo $\mathrm{XX}$, se ha llamado la atención sobre la histórica inclinación de la antropología a primar el parentesco sobre las bases sexuales que lo producían (Dayan-Herzbrun, 1991; Nieto, 2001, pp. 142-143). Dicho de otra forma, el parentesco asumía (sin mencionarlo) una sexualidad subyacente, pero pautada, que tomaba cuerpo en ligazones parentales, también pautadas e institucionalizadas; hablando en términos teóricos, el parentesco venía a ser el resultado social de una función orgánica que, por ser función, pero sobre todo por ser función orgánica, no requeriría un examen específico por parte de las ciencias sociales (Nieto, 1997). Y, dicho también de otra forma, la sexualidad venía a entenderse dentro de un ámbito institucional. Como muestra, el título que dio Lévi-Strauss a las conferencias que impartió en Japón en 1986 (2012): "Tres grandes problemas contemporáneos: la sexualidad, el desarrollo económico y el pensamiento mítico", donde la sexualidad se interpreta a través de la organización familiar y social, concretamente a través del parentesco, para interrogarse finalmente sobre las consecuencias morales y sociales de la disociación de la sexualidad y la reproducción. La antropología suponía, sobre la base de estudio de pequeñas comunidades, que sexualidad y relación parental eran lo mismo, de manera que hablar de sexualidad era una especie de licencia retórica. Si en dichas sociedades no había disociación entre los dos ámbitos (sexualidad y filiación) no era de interés estudiar sexualidades sino la lógica social del parentesco por encima de la carnalidad sexual. En tal perspectiva, un estudio de sexualidades se consideraría una curiosidad antropológica que en un plano científico no aportaría nada a la comprensión de la naturaleza humana.

En los planes de estudio clásicos se estudiaba antes una "antropología del parentesco" (de la familia, del matrimonio, de la exogamia...) que una "antropología de la sexualidad", por mucho que -a todos los efectos- ésta estuviera, solapadamente, en la raíz misma de aquella (y, al mismo tiempo, desenraizada del parentesco). 


\section{De la Emergencia Teórica y Académica de la Sexualidad y de la Crisis del Parentesco}

Paradójicamente, el transcurrir del tiempo ha puesto entre interrogantes el ámbito del parentesco y ha visibilizado el ámbito de la sexualidad; o, si se prefiere, se ha pasado del parentesco en la mente (como hecho social y aritmético) al parentesco en el cuerpo; y de la sexualidad en el cuerpo, entendido como organismo y como individualidad teóricamente denostada, a la sexualidad en el cuerpo como individualidad teóricamente emergente y como construcción social.

El cuestionamiento del parentesco clásico se ha formalizado porque la tecnología y otros cambios sociales (adopciones y parentescos que podríamos llamar "de cuerpo") están obligando a revisar los conceptos clásicos y demostrando que las ligazones no biológicas crean también relaciones de parentesco ${ }^{1}$, lo que anima a preguntarse qué es el parentesco y cómo se institucionaliza cuando la sexualidad no está de por medio, es decir, cuando no hay vínculos de esperma y óvulos. En cuanto a la sexualidad, su repunte teórico guarda relación con la emergencia de los "sujetos" (Touraine, 2005) en sociedades muy pautadas normativamente y con la progresiva incorporación teórica del cuerpo como objeto de estudio de las ciencias sociales, en una interesante "batalla" entre las ciencias biomédicas y las sociales que, en términos históricos, acaba de ponerse en marcha. Ahora hay más sexualidad donde antes había más institución; hay más cuerpo.

El correlato académico de esta reestructuración de las posiciones que ocupan en la realidad contemporánea tanto las estructuras del parentesco como la sexualidad lleva mostrándose al menos tres décadas si nos atenemos a la progresiva inclusión del "cuerpo" en los estudios y teorizaciones de sociólogos y antropólogos y a la crisis de las instituciones tradicionales familiares y parentales entre ellas- y, en general, del estructuralfuncionalismo. Digamos que, actualmente, se han equilibrado los estudios de institucionalización y los de sexualidad. No tanto en los actuales planes de estudio como en publicaciones científicas que, desde las ciencias sociales, reivindican la sexualidad como un objeto legítimo de estudio allí donde la medicina o la psiquiatría tenían el monopolio del saber. Ahora las ciencias sociales se enfrentan con las ciencias biológicas por el cuerpo y por la sexualidad, siendo la sexualidad un concepto heredado que resulta difícil de 


\section{De la Calle Valverde - Sociología de la Diversidad}

aprehender y sobre el que resulta complicado trabajar. Como resultado, el parentesco ha perdido visibilidad académica, tanto en instituciones universitarias (de Gran Bretaña y EEUU) como en investigaciones y publicaciones científicas (Collard, 2000). En el plano estrictamente académico, aún han tenido que pasar algunos años más para que esa teorización creciente sobre el cuerpo y, más concretamente, sobre la sexualidad, alcanzara un espacio entre otras disciplinas clásicas. Aunque, como toda disciplina emergente a razón de la crítica de lo clásico, es probable que se vea desplazada a los márgenes y vea ralentizada su incorporación al corpus de conocimiento antropológico por razones de tipo institucional y académico (Bazin, Mendes-Leite y Quiminal, 2000).

\section{Antropología, Sociología y Sexualidad en los Planes de Estudio Universitarios en España}

Este proceso, que se acelera más en unas sociedades que en otras, asoma en España en la década de los 90 (Guasch y Viñuales, 2003; Nieto, 2004), cuando tanto la antropología como la sociología de las sexualidades comienzan a incluirse en los planes de estudio universitarios. En 1983, Iglesias de Ussel nos ofrecía una visión condensada de la situación de los estudios sobre sexualidad en España, en perspectiva histórica y adelantada; para la fecha, afirmaba que "hoy no existe ningún tipo de incorporación institucional de los estudios sobre sexualidad en la Universidad española" (Iglesias de Ussel, 1983, p. 107). Poco después de esta afirmación, entre 1985 y 1989, José Antonio Nieto impartió en la Universidad Nacional de Educación a Distancia (UNED), y como enseñanza no reglada, una "Antropología Sexual" desde una perspectiva estrictamente antropológica. A partir de esta experiencia se construyó un proyecto de Master de la mano del profesor Nieto.

En 1989, a propuesta de este profesor, la UNED aprobó por primera vez en la universidad española un programa de estudio sobre sexualidad, con titulación propia (enseñanza no reglada), que se constituyó en un Máster en Sexualidad Humana que se puso en marcha en 1990. El máster se impartía en 2 cursos académicos con 10 asignaturas. Su contenido era multidisciplinar pero se subrayaba la relevancia de la organización sociocultural de la sexualidad (códigos, pautas, comportamientos, 
pensamientos...). Esa perspectiva multidisciplinar fue un requisito de las sucesivas comisiones encargadas de aprobar el proyecto.

Las 5 asignaturas del primer curso fueron las siguientes: Cultura $y$ Sociedad en las prácticas sexuales (aproximación socioantropológica)/Bases anatómicas y fisiológicas de la sexualidad y la reproducción humana (aproximación clínica)/Educación sexual (aproximación pedagógica)/Sexualidad humana. Estudio y perspectiva histórica (aproximación histórica)/ Biología y psicofisiología de la conducta sexual (enfoque biológico). Las 5 del segundo curso: Género y Sexualidad (perspectiva de género)/La sexualidad en la sociedad contemporánea. Lecturas antropológicas/ Sexualidad y sexismo/ Epidemiología y prevención de las enfermedades de transmisión sexuall Terapia sexual. Las 10 asignaturas generaron sus propias publicaciones como material de estudio. El máster estuvo vigente hasta el año 2000.

Haciendo excepción de lo apuntado, las investigaciones sobre sexualidad en España seguían una tónica de invisibilidad institucional 10 años después de las afirmaciones hechas por De Ussel (Guasch, 1993).

Desaparecido el máster, el profesor Nieto siguió interesado en dar continuidad académica a la perspectiva socio-cultural/antropológica de la sexualidad, siendo el artífice de la introducción de la disciplina Antropología de la Sexualidad en España y en enseñanzas regladas ${ }^{2}$. Así, de la mano de José Antonio Nieto, traductor de etnografías sexuales para un público poco familiarizado, la sexualidad se nos presentó desde la mirada de la antropología social y cultural, más allá de la mirada sexológica (Weeks, 1993), tan corriente allí donde la psiquiatría y la medicina (y la moral) cabalgan casi juntas. Su aparición (en la UNED) fue, sin duda, un acontecimiento académico en el contexto de la universidad española. El hecho tuvo lugar en el curso 2003-2004, tras ponerse en marcha la Licenciatura de Antropología Social y Cultural en dicha universidad. Su plan de estudios se publicó en noviembre de 2002 en el Boletín Oficial del Estado (BOE) y contemplaba, inicialmente, una asignatura denominada "Sexualidad Humana" que, después de vencer tensiones académicas administrativas, se concretó en algo más específico: una "Antropología de la Sexualidad" que se desligaba de la medicina, de la psiquiatría y de la sexología, y que aportaba argumentos, reflexiones y, en general, material para un acercamiento social y cultural a la sexualidad. Es en este punto y sentido en el que hay que 
entender esta irrupción como acontecimiento: la institucionalización en España de un estudio de la sexualidad en clave social y cultural.

\section{Una Asignatura-Acontecimiento en la Historia de la Universidad Española}

La UNED es la primera universidad en la que se imparte una "Antropología de la Sexualidad", en el contexto de una Licenciatura, Diplomatura o Grado. Como se apuntó, desde el curso 2003-2004 la asignatura se incluye en los planes de estudio de la Licenciatura de Antropología Social y Cultural, que se imparte desde la Facultad de Filosofía.

La acogida de la nueva licenciatura fue notable a lo largo de los primeros cursos. Al tratarse de una licenciatura de segundo ciclo $^{3}$ y facilitándose el acceso desde otras áreas de conocimiento, la matriculación alcanzó niveles considerables desde el primer año de implantación, por encima de los 5000 estudiantes el primer año de implantación y por encima de los 70002 cursos académicos más tarde, según aseguraba el decano de la Facultad de Filosofía, Manuel Fraijó, en las sucesivas Guías de la Licenciatura que se publicaron, lo que rompió las mejores expectativas de la facultad que creó la licenciatura. El primer curso de la licenciatura estaba compuesto por 7 asignaturas troncales y obligatorias: Antropología del Parentesco, Antropología Cognitiva y Simbólica, Etnografía y Técnicas de Investigación Antropológica, Historia de la Antropología, Métodos Estadísticos en Antropología Social, Geografía Humana y Demografía y Estructura Social. El segundo curso por 6: Antropología Económica, Antropología Política, Hominización, Etnología Regional, Estadística Aplicada a las Ciencias Sociales y Filosofía de las Ciencias Sociales. Además, el alumno disponía de 15 asignaturas optativas para completar el número de créditos necesarios para la obtención del título (más las asignaturas de libre configuración). Esta fue la estructura final, algo cambiante en relación a la que se publicara años atrás en el BOE.

La Antropología de la Sexualidad se incluyó en el plan de estudios como asignatura optativa y ya desde el primer curso tuvo niveles de matrícula que superaban, sobradamente, los 1000 alumnos (Gráfico 1) ${ }^{4}$. El año en el que se inauguraba la licenciatura, el interés de los matriculados también se orientó hacia esta optativa que alcanzó la cifra record de 1869 matriculados; a partir 
de aquí la matrícula descendió, quedando siempre por encima de 1000 alumnos, excepto cuando comienza el proceso de extinción de la licenciatura y de adopción de los nuevos grados como resultado de la puesta en marcha en España del Plan Bolonia y el proceso de adaptación al Espacio Europeo de Educación Superior, lo que tuvo lugar en el curso 2010-2011 para el caso que estamos comentando.

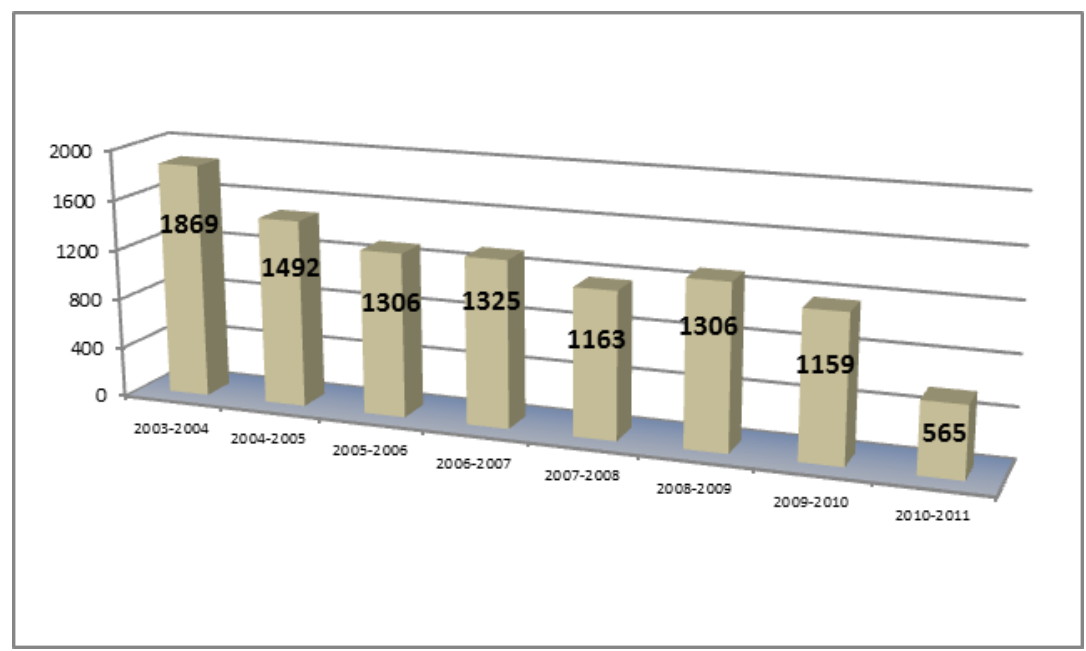

Gráfico 1. Número de alumnos matriculados por curso académico en Antropología de la Sexualidad. Período 2003-2004 / 2010-2011

Fuente: Actas de cada curso académico

A lo largo de todos los años de su existencia, la asignatura tuvo el mayor número de matriculaciones de todas las asignaturas optativas de la licenciatura, con una diferencia ostensible. La implantación progresiva de los nuevos grados hizo descender, lógicamente, los niveles de matriculación. El nuevo Grado en Antropología Social y Cultural se imparte desde el curso 2010-2011. Desde ese curso académico los alumnos ya están migrando al nuevo plan de estudios en donde la "Antropología de la Sexualidad" ha desaparecido como asignatura. El último curso con matriculaciones es el curso 2013-2014, para el que se contempló una única prueba de gracia a realizar en el mes de febrero de 2014. En estos años el Equipo Docente estuvo formado por José Antonio Nieto -desde 2004 hasta la extinción de la 
asignatura- y por el autor de este artículo -desde 2004 hasta 2011-.

El proceso de "sexualización de la antropología", -"sexing anthropology"- que Herdt (1999) reconocía finalizando el siglo XX, ha tenido, como se ve, tímidos avances en nuestro país pero significativos para impulsar la dimensión académica de la Antropología de la Sexualidad en España (Nieto, 2004).

\section{Un Acontecimiento Intelectual para los Alumnos de la Licenciatura}

Desde el primer curso de implantación de la asignatura en la Licenciatura de Antropología Social y Cultural se observó que los alumnos estaban experimentando una especie de apertura intelectual, que fue descrita e interpretada en un artículo específico (De la Calle, 2011) cuyas conclusiones se abrevian en este apartado.

El texto de estudio de la asignatura (Nieto, 2003) era un compendio de artículos, más o menos recientes, que su coordinador tradujo con el objetivo expreso de crear un volumen específico para la asignatura. El texto definitivo no era un manual al uso sobre sexualidad, sino una panorámica transcultural de la sexualidad desde una perspectiva antropológica. Diversos autores escribían sobre diversas temáticas que formaban parte de una discusión contemporánea sobre la sexualidad; los diferentes capítulos no guardaban entre sí una ligazón digamos "didáctica”, sino (acaso) una ligazón más bien teórica, de forma que el paso de un capítulo al siguiente no respondía a un sentido lineal sino más bien a un sentido reflexivo. Los autores que componían con sus artículos el texto base de la asignatura miraban la sexualidad con ojos antropológicos y eso creaba un nexo de unión suficientemente fuerte como para conformar un texto de licenciatura coherente a la vez que novedoso y que, por descontado, introducía al alumno de lleno en algunas de las múltiples discusiones contemporáneas sobre la sexualidad humana.

Desde la familia en perspectiva transcultural, hasta el género, la identidad sexual, comportamientos sexuales a los que no estamos acostumbrados, etc... cuyo objetivo era hacer rodar la rueda del pensamiento en los alumnos para acercarse a la sexualidad no como a algo natural, sino construido o, al menos, algo que exige una reflexión ulterior antes que una serie de comentarios dogmáticos. 
Sin embargo, el deseado esfuerzo exegético de los alumnos quedó en agua de borrajas en buena parte de los casos, lo que era una muestra del impacto que tuvo la asignatura y de las dificultades para dotar de sentido las sucesivas lecturas. Algunos alumnos dejaban constancia en sus hojas de examen del impacto intelectual que les había causado la lectura; chocaba con tradicionales formas de entender la sexualidad; utilizaban el concepto de "etnocentrismo" para describir su postura intelectual precedente y aconsejaban un aperturismo reflexivo para captar el sentido de la sexualidad en un plano universal y, a la vez, más allá de todo determinismo universal.

En el aspecto práctico, los exámenes se resolvían a menudo con el uso de analogías entre lo que leían y sus experiencias o conocimientos precedentes, y este era otro indicativo del impacto intelectual y de la necesidad de dar sentido a la lectura. Es decir, los alumnos traían a su mundo de conocimientos lo que leían en el texto mediante el uso de analogías. Identidades sexuales en otros lugares del planeta les sugerían identidades sexuales en nuestra sociedad, comportamientos sexuales en otras culturas les sugerían comportamientos sexuales en la nuestra...

Esto no enriquecía los exámenes, pues no se trataba de examinar la sexualidad de otras áreas culturales con el prisma de la nuestra, sino de mirarlas en otros contextos culturales donde realmente toman sentido. Este esfuerzo intelectual, separar lo que parece unido por una especie de moraleja oculta en todo el texto y que atraviesa todos los capítulos (de tipo moral, por ejemplo, "nuestra sexualidad es mala, la de ellos es más permisiva"), no se concretaba más que en algunos alumnos especialmente preparados y con conocimientos previos, que miraban el texto con ojos teóricos más que normativos o morales; el capítulo introductorio (correoso para la mayoría de alumnos) daba una pista para poder entender el texto completo de esta forma; situaba la discusión contemporánea en materia de sexualidad en torno al cuerpo y se hacía eco de los posicionamientos divergentes de la biomedicina y la antropología sobre la sexualidad. La batalla por el cuerpo era reciente y las ciencias sociales (especialmente el constructivismo) llevaban algunos años asaltando la tradicional fortaleza de las ciencias que llamamos duras. A nuestro pesar, esta sugerencia teórica del primer capítulo no fue explotada por los alumnos en condiciones suficientes como para poder entrar a examinar el texto con una postura interpretativa adecuada. La exégesis esperada no fue tal. 
Teóricamente, con el objetivo de interpretar el comportamiento reflexivo de estos alumnos, se aplicaron los conceptos de estructura y acontecimiento para concluir que algunos alumnos habían experimentado un "acontecimiento intelectual" que chocaba con su estructura de conocimientos y nociones sobre sexualidad. El objetivo del artículo, ya referido (De la Calle, 2011), era ofrecer a los futuros alumnos las herramientas necesarias para afrontar la asignatura con rigor.

\section{Antropología de la Sexualidad en la Diplomatura de Trabajo Social de la UNED}

Hasta el momento se ha hecho referencia a la impartición desde el curso 2003-2004 de una Antropología de la Sexualidad de base social y cultural en la Licenciatura de Antropología Social y Cultural de la UNED. En el curso académico 2004-2005, se implantaba la Diplomatura de Trabajo Social en dicha universidad, adscrita inicialmente a la Facultad de Ciencias Políticas y Sociología. En la diplomatura se contemplaba la asignatura "Antropología Social" como asignatura troncal que, (fruto de negociaciones entre departamentos y facultades), fue adscrita al Departamento de Sociología I de la Facultad de Ciencias Políticas y Sociología, e impartida nuevamente por el mismo Equipo docente que estaba impartiendo Antropología de la Sexualidad en la Licenciatura de Antropología Social y Cultural.

El ED diseñó sus contenidos a partir de 2 textos de naturaleza dispar. El primero de ellos una obra norteamericana de antropología general, de amplia difusión en el ámbito universitario de nuestro país: Antropología Cultural de Phillip Kottak. El segundo de los textos era el mismo que se estaba utilizando en "Antropología de la Sexualidad", el texto de Nieto ya mencionado. En este sentido también se impartió "Antropología de la Sexualidad" en la Diplomatura de Trabajo Social hasta su extinción y reconversión en Grado. La implementación de Trabajo Social aportó a la UNED un bueno número de matriculaciones y dado que la asignatura era troncal, de nuevo hubo un gran número de matriculados.

La virtualización de las asignaturas ya era corriente en esta universidad y permitió al Equipo Docente seguir a través de los foros las impresiones causadas por la lectura de ambos textos. 


\section{RISE - International Journal of Sociology of Education, 5(2) \\ Acontecimiento Intelectual en los Alumnos de la Diplomatura de Trabajo Social}

En la asignatura Antropología Social de Trabajo Social, algunos alumnos manifestaron a través del foro la inquietud reflexiva que les suscitaba la lectura del libro Antropología de la Sexualidad y Diversidad Cultural, que se complementaba con un recopilatorio y clásico libro de texto norteamericano de Kottak. Mientras que el segundo libro es muy didáctico y pedagógico (las cosas son lo que son) el primero invita a romper las prenociones que tenemos sobre lo que es y no es en materia de sexualidad. Un alumno de Trabajo Social lo expresó así en una intervención en el foro:

Estimado profesor, la verdad es que es un placer contar con tanto detalle en cuanto a la preparación de la asignatura, aunque a mí me surge una duda, ahora que estoy siguiendo las indicaciones para prepararla leyendo ambos libros y en vista al examen, no sé si podrá aclarármelo, pero veo que el libro de Kottak es como más técnico, hay más conceptos, definiciones, acompañados de ejemplos etnográficos, clarificadores y gratificantes y muy pedagógicos, y sin embargo el libro de Nieto es una especie de compilación de textos de diferentes autores que llevan más a la reflexión...

Tras esta reflexión inicial, el alumno expresó su parecer tras la lectura de los textos, que recojo en su totalidad a continuación:

(...) Muchas gracias por anticipado, y quiero aprovechar para hacer explícita mi reciente pasión por esta asignatura y en general por la antropología, que me está haciendo cuestionarme muchos de los valores y principios que tenía, es más creo que en muchos aspectos se me han derrumbado, el conocer otras culturas nos hace muchísimo más racionales como decía la reciente fallecida y magnífica escritora y docente Josefina Aldecoa, de la que soy un gran seguidor.

(En respuesta a esta intervención en el foro, solicité al alumno que desarrollara más los argumentos a la vez que reconocí la diferencia de estilos entre uno y otro libro de texto)

En cuanto a su interés docente por cómo me han impactado las 
lecturas sobre todo del libro de Nieto, creo que daría para un largo e interesante debate, pero trataré de concretar. Cuando comentaba que muchos de mis principios se me han derrumbado, me refiero a cómo la sexualidad es también algo que se construye en cada sociedad o incluso en el ambiente en el que nos socializamos, de manera que nosotros los "occidentales", a los que nos han educado con unos valores y principios muy concretos, (con enorme influencia de la moral y valores católicos) y que yo al menos no me había cuestionado nunca, es decir nunca había reflexionado sobre la sexualidad, como algo que se construye socialmente. Es cierto que todos tenemos una postura respecto a la homosexualidad, lo transexual, incluso en temas legales como la aprobación del matrimonio gay, pero más allá de esta forma de cuál sea esa postura, en la que yo me consideraba una persona abierta, tolerante con esos temas, creo que no es una cuestión de ser más o menos tolerante con la sexualidad de los demás, sino que deberíamos despojarnos de todos los prejuicios en los que nos han educado, y ver que la sexualidad es algo tan personal, y que va muchísimo más allá de la implicación de hombre- mujer, o heterohomo-bis-sexual, que es muchísima más amplia y abarca muchos más aspectos sobre todo después de leer los textos sobre los hijras de la India.

No sé si me he explicado bien, aunque reconozco que soy demasiado conciso y que es un tema tan interesante como largo, además de que aún no he "digerido" por decirlo de alguna manera, lo ignorantes que podemos llegar a ser, creyéndonos el ombligo del mundo, y que tenemos mucho que aprender de otras culturas de personas que consideramos "salvajes" por lo que agradecería al resto de compañer@s que tengan algo que aportar que lo hagan, podríamos construir un emocionante debate, sobre todo sobre la sexualidad desde el punto de vista de la antropología, que he de reconocer que cada día me entusiasma más, hasta el punto de que quiero terminar trabajo social, y pasarme al mundo de la antropología, cuando mi intención era la sociología, ciencias al fin y al cabo "hermanadas".

Las palabras de este alumno no son una excepción. A título ilustrativo, estas son otras 2 opiniones extraídas del foro en el que solicitó a los alumnos que lo desearan que dejaran plasmadas sus reflexiones:

Al leer los comentarios de mis compañeros y al notar su interés por el 
impacto que está causando el estudio de esta asignatura en cada uno de nosotros también quiero dejar el mío.

Me ha ocurrido lo mismo que a mi compañero, al crecer en una sociedad occidental, cargada de moralidad, uno piensa que nuestra mirada sobre la sexualidad es la correcta, y no se para a pensar en que existen otras formas de relacionarse sexualmente.

Pensamos que tenemos la "verdad absoluta" y no aceptamos, o no nos interesa aprender, de otras formas de relaciones. Sinceramente, yo nunca me identifiqué con esto pero sin embargo, he de admitir, que me ha influido mucho en mi forma de vida. La lectura del libro de Nieto me ha hecho abrir los ojos e ir un poco más allá de lo que normalmente me rodeo. Incluso he llegado a imaginar mientras leía cómo se hubiera desarrollado mi vida si hubiera crecido en otro tipo de culturas, cómo me comportaría, cuál sería mi forma de relacionarme, etc.

Al finalizar la lectura he llegado a la conclusión de que muchas culturas están más avanzadas que la nuestra. Por poner un ejemplo, en lo que se refiere a diferencia de género tomo la sociedad Huaorani, en la que las diferencias de género se minimizan, valorando mucho más la autonomía personal y el igualitarismo, dónde la violencia doméstica está totalmente ausente de las relaciones sociales. Sin embargo, su forma de relacionarse eróticamente entre todos los miembros de la sociedad sería muy cuestionada por nuestra sociedad e incluso rechazada. Se los trataría de "bárbaros", pero yo pienso, ¿quién es más "bárbaro" aquél que maltrata a su pareja física y psicológicamente, que no le permite progresar en la vida sólo por formar parte del grupo del "sexo débil", que durante siglos ha oprimido a un grupo de personas sólo por considerarlas inferiores ( sin ninguna base) o aquellos, como los huaorani que simplemente son libres en sus relaciones, que practican la sensualidad como expresión corporal de la participación en las relaciones, como una búsqueda de la cohesión grupal....?

En suma, yo soy otra alumna que también se está planteando terminar Trabajo Social y continuar con estudios de Antropología. Me apasiona la gente y me encanta aprender no sólo de lo que siempre he conocido, sino ir más allá. El estudio de esta asignatura me ha mostrado algo más que no conocía y ha despertado un gran interés en mi de profundizar estos nuevos conocimientos que estoy adquiriendo. 
Acabo de terminar de leer el temario y me ha parecido muy interesante. Creo al igual que algunos de mis compañeros que es una profesión apasionante. A mi parecer es una forma de dar a conocer otras culturas, acercarnos a ellas pero enseñándonos a no juzgarlas. Creemos ¡bueno! nos hacen creer que nuestra cultura es la correcta y que las que son diferentes son una especie rara, atrasada, sin educación ni valores. Referente al tema de la sexualidad, creo que hay otras culturas que están mucho más avanzadas que la nuestra en el tema de la diversidad de género como es el caso de los hijra en la India o la sadhim. Como última reflexión quiero decir que desde hace unos años han empezado a hablar de la globalización, de la multiculturalidad ,......pero creo que para que la gente de a pie empiece a comprender realmente lo que son estos conceptos habría que enseñarles la existencia de otras culturas, su forma de pensar, vivir, amar....y eso empieza por llevar todos estos temas a las aulas.

Ya se constató algo similar en los estudiantes de la asignatura "Antropología de la Sexualidad" de la Licenciatura de Antropología Social y Cultural. Más significativo, tal vez, en alumnos de Trabajo Social, dada su orientación práctica. Es decir, en Trabajo Social constatamos las mismas estrategias intelectuales para suavizar el impacto de la lectura del texto, las mismas herramientas conceptuales para interpretar a los "otros" y al "nosotros" y el elogio de las culturas distantes frente al desprecio de las culturas próximas.

Y de la misma forma que se aclaró entonces conviene reeditarlo de nuevo: ni para todos los alumnos la lectura supuso un acontecimiento intelectual (o al menos de la misma intensidad) ni todos reaccionaron de la misma forma a la novedad de los contenidos.

\section{Las Negociaciones que Trajo Bolonia y el Fin de la Antropología de la Sexualidad}

La etapa duró 11 cursos académicos en el caso de la "Antropología de la Sexualidad" impartida en la licenciatura y algo menos en el caso de "Antropología Social" de la diplomatura de Trabajo Social. La implantación de los nuevos grados sometió la estructura de los estudios universitarios a revisión. Por ser más justo con los hechos, los planes de estudio fueron sometidos a negociación. Entramos entonces en cuestiones de índole 
jerárquica y de poder, en donde aquellas personas y departamentos que tienen más peso institucional hacen valer sus posiciones y primar sus intereses sobre los del resto, aunque en el resto existan razones suficientes para defender un proyecto académico.

De tal guisa, la "Antropología de la Sexualidad" desapareció en el nuevo Grado de Antropología Social y Cultural y la "Antropología Social" de la Diplomatura de Trabajo Social fue adscrita al departamento de Antropología de la Facultad de Filosofía cuando se implementó el nuevo grado en Trabajo Social.

Aunque la implantación del Plan Bolonia, -con su insistencia en la práctica y en el saber aplicado, y su inclinación a empresarializar la universidad en perjuicio de los clásicos saberes teóricos- no facilita el camino a las ciencias sociales, dicho plan no es el causante de la pérdida de visibilidad estructural de la asignatura; más bien es la excusa para recolocar y reestructurar lo que se consideraba descolocado. Y, sin embargo, la aplicación práctica de la "Antropología de la Sexualidad" no puede ser obviada en la sociedad contemporánea. El redescubrimiento antropológico de la sexualidad desde el último cuarto del siglo pasado (Vance, 1991) -pese a las sorprendentes advertencias de Malinowski en los años 30 del mismo siglo sobre el "exceso de sexo" en las investigaciones (Nieto 2011, p.186)es un indicador de su creciente visibilidad y posicionamiento para abordar la materia. El auge progresivo que en nuestra sociedad han tenido y tienen los trabajadores sociales son un argumento suficiente para considerar la perspectiva antropológica de la sexualidad como primordial, pues no se puede actuar socialmente sin conocer sobre qué se está actuando; sea prostitución o SIDA, heterosexualidad y relaciones de pareja, transexualidad y cuestiones de identidad..., la antropología socio-cultural (además de otras disciplinas afines) aporta un bagaje teórico para trabajar en la práctica con fundamento.

En la práctica, la libertad de cátedra permitió que la "Antropología de la Sexualidad" mutara en otra cosa y en otro lugar. En la discusión interna sobre el nuevo Grado de Sociología en la Facultad de Ciencias Políticas y Sociología de la UNED se acogió de buenas maneras una "Sociología de la Diversidad" propuesta por el mismo Equipo Docente que había impartido la asignatura que se menciona. La "Sociología de la Diversidad" fue la unión de dos cadáveres: uno teórico ("Conflicto Social y Conducta Desviada"), y 
otro institucional (“Antropología de la Sexualidad”).

\section{De Conflicto Social y Conducta Desviada a la Sociología de la Diversidad}

La "Antropología de la Sexualidad” desapareció como tal del nuevo Grado en Antropología Social y Cultural, pero mutó en una nueva asignatura. Parafraseando: "La Antropología de la Sexualidad" no se destruye, solo se transforma. Fue así como a su ocaso en el Grado en Antropología Social y Cultural -que era su "espacio natural"-, sucedió su amanecer en el Grado en Sociología. En la discusión sobre la estructura y contenidos del Grado en Sociología se propusieron muchas y especializadas sociologías, y viejas y revisitadas asignaturas (que finalmente fueron las que triunfaron en mayor proporción). En las comisiones formadas para discutir los nuevos grados se aceptó una novedosa "Sociología de la Diversidad" propuesta por el profesor José Antonio Nieto; que finalmente se incluyó en el nuevo grado, en tercer curso y como asignatura obligatoria. La peculiaridad de esta asignatura es que conjugaba una destrucción más un renacimiento. La destrucción de Conflicto Social y Conducta Desviada tenía fundamento teórico, que fue desarrollado densamente en el nuevo libro de texto escrito con el objetivo de cubrir los contenidos de la nueva andadura (Nieto, 2011).

¿Por qué este cambio? ¿Por qué se transforma la sociología de la desviación, la (sociología de la) conducta desviada en sociología de la diversidad? Sencillamente, porque desde hace ya treinta y cinco años la sociología de la desviación, como tal denominación, ha venido cuestionándose. Y ello se ha debido a que la desviación con respecto a normas sociales, efectuada por sujetos sociales que por medio de acciones quiebran estructuras normativas, queda diluida conceptualmente. Las conductas desviadas individuales que propician la desviación, contempladas desde la perspectiva de los derechos de la ciudadanía, quedan desdibujadas. Dejan de tener el peso en oro teórico establecido en valores absolutos, en que venían fundamentándose (Nieto, 2011, p. 30).

En la historia de la antropología (al igual que en la historia de la sociología), las subjetividades han tenido poca atención (Nieto y De la Calle, 
2013); los planteamientos teóricos clásicos que tuvieron mayor impacto respondían a cuestiones de tipo estructural, funcional, evolucionista..., en donde el individuo solo aparece como un reflejo estructural, una posición social, un status, un rol... Fuera de esta visión teórica del individuo hay pocas herramientas para interpretar y entender la subjetividad desde la antropología o desde la sociología sin tener que desplazarse de la disciplina e invadir otras áreas de estudio como la psiquiatría, con su tendencia a considerar desviación todo lo que se salga de la norma social en materia de identidad subjetiva.

La explosión de los individuos tiene lugar en el último cuarto del siglo XX. Se pasa de la visibilidad de las estructuras a la visibilidad de los individuos. Las tomas de decisiones, el cuerpo, las emociones y todos aquellos "componentes" de subjetividad empiezan a incorporarse a las investigaciones antropológicas (y sociológicas). La explosión de las individualidades pone en entredicho la conformación de los sistemas sociales, de las estructuras e instituciones comunitarias, de las normas y los valores tradicionales...

El nuevo texto para "Sociología de la Diversidad" es una reivindicación de la diversidad de los sujetos. Incluso acotando la diversidad al universo de las identidades sociales, la amplitud de esta sociodiversidad es tal que obligó al responsable de la nueva asignatura a acotar, aún más, los contenidos que formarían la materia de estudio. Siguiendo esta idea, se decidió por confeccionar una obra dedicada a la diversidad en materia de identidad sexual o de género.

\section{De la Antropología de la Sexualidad a la Sociología de la Diversidad.}

Si "Conflicto Social y Conducta Desviada" desapareció del nuevo plan de estudios, el renacimiento correspondió a la "Antropología de la Sexualidad", especialmente interesada en la diversidad sexual (de conductas, identidades, formas de pensar...) de base social y cultural. De esta manera se planteó una Sociología de la Diversidad incipiente, centrada en la complejidad identitaria contemporánea y, concretamente, en las identidades relacionadas con la sexualidad.

...Este proyecto docente sobre sociodiversidad, como tal, es innovador en los planes de estudio de las facultades de sociología (al 
menos en la universidad española). Como innovador, es un proyecto que se encuentra en su fase inicial y, por tanto, en estado embrionario. Embrión que con el tiempo -espero- irá metabolizando ideas y musculando conceptos, haciendo corpus, para lo cual tendrá necesariamente que desarrollar sus propuestas epistemológicas. A las limitaciones epistemológicas, propias de las propuestas novedosas, hay que sumar las limitaciones de su configuración académica de asignatura cuatrimestral (que a su vez viene configurada por las estrecheces pedagógicas del denominado Plan Bolonia). Todo ello implica acotar, para que sirva de referencia al alumno, el campo de estudio de la sociología de la diversidad. Como en sí misma, la sociodiversidad pudiera ser inagotable, la acotación se lleva a efecto mediante una muestra, de las muchas posibles: la sexualidad... (Nieto, 2011, p. 27).

Afinando aún más, la sociodiversidad se acotó y aplicó al universo de las identidades transexuales. Del texto de la asignatura que se impartía en la Licenciatura de Antropología Social y Cultural se tomó el capítulo introductorio y se añadieron un conjunto de apartados orientados a entender e interpretar la identidad transexual en clave social y cultural. Pues, tras lo que se conoce y entiende como "transexualidad" se esconde una enorme variedad identitaria:

Mientras en el Arco [Barcelona] sabía, por la actividad a que nos dedicábamos, que las trans, por diferentes que fuésemos, pertenecíamos a un mismo grupo humano, por el colectivo [CTC, Colectivo de Transexuales de Catalunya] desfilaba una humanidad tan variada que a veces me preguntaba si teníamos algo en común, aparte de decir que nos considerábamos transexuales, sin que en muchos casos nada respaldase esa afirmación. No existe ningún acuerdo entre quienes estudian la transexualidad ni sobre su etiología ni sobre su clasificación. La variedad entre los individuos que dicen formar parte de ella puede ser tan grande que, aparte de su no verificable autoproclamación, es difícil encontrar algún parecido entre ellos (Mejía, 2006, p. 136).

El texto pertenece a una persona transexual que tiene el valor añadido de ser la primera persona transexual que en España lee una tesis doctoral en el 
área de antropología... y sobre transgenerismos.

Si cruzamos algunas variables como las que siguen, -cada una de ellas con sus componentes-...

--Fisiología y anatomía original (morfología del cuerpo en el nacimiento)

-Fisiología y anatomía de destino -si la hubiera- (morfología corporal deseada por la persona trans)

-Prácticas de transformación del cuerpo -si las hubiera- (CRS -Cirugía de Reasignación de Sexo-, hormonación...)

--Orientación sexual (homosexual, bisexual, heterosexual, indistinta, transexual...)

--Sentimiento de identificación (autoidentificación)

...obtendremos como resultado una inmensa variedad de individuos que difícilmente se dejan clasificar bajo una misma etiqueta (Nieto, 1998, p. 21).

Bajo un concepto (más o menos discutido) como "transexualidad" se esconde una enorme diversidad social, un mosaico de individuos que tienen en común su marginación social -igualdad en la diferencia- que los asocia en comunidades políticas en defensa de sus derechos.

\section{La Comunidad Transgenérica como Archipiélago de Identidades. Del Sujeto-Cuerpo a la Comunidad y los Derechos Políticos}

Hasta comienzos de los 90 del siglo XX, las personas norteamericanas de géneros cambiantes, y concretamente las transposiciones de varón a mujer, se agrupaban en travestidos y transexuales, cuya clasificación simple respondía a los siguientes criterios:

Los transexuales de varón a mujer se definían a sí mismos con un criterio básico de deseo de reasignación hormonal y quirúrgica (...). Si una persona no estaba convencida del todo a someterse a cirugía, entonces esta persona era de facto un travestido. A los travestidos se los describía como varones heterosexuales (varones atraídos por las mujeres) que se veían impelidos a travestirse pero que no eran realmente mujeres. Si estos individuos tenían una identidad femenina parecía razonable que se sometiesen a una operación de cirugía -sin disculpas- (Bolin, 2003, p. 253). 
Pocos años después esta dicotomía (travestidos/transexuales) era criticada "desde dentro" de las asociaciones que tendían a englobar dichas identidades en una especie de continuum de variantes de género caracterizado por la diversidad en su composición, por la estigmatización desde fuera y por la conciencia de la necesidad de su agrupamiento con fines políticos, esto es, con vistas a la consecución de derechos. Se discutía el concepto adecuado para poder dar cabida a la diversidad identitaria, teniendo en cuenta que dicha discusión valoraba con conocimiento de causa el débito histórico de las personas trans a las taxonomías de inspiración médica o psiquiátrica. Así, en un texto de 1994, Anne Bolin describía el surgimiento en EEUU de la comunidad transgenérica:

Gracias a recientes investigaciones resulta evidente que ha habido un movimiento en el que las personas con identidades de genero transpuesto se han organizado como parte de una comunidad más amplia, un grupo interno más grande, que se enfrenta a problemas similares de estigmatización, aceptación, trato, etc. Este reconocimiento de las semejanzas, impulsado por una conciencia política creciente de las organizaciones de género, ha facilitado el surgimiento de nuevas opciones de género, tales como la "transgenerista". Transgenerista es un término comunitario que denota parentesco entre aquellos con identidades de variante de género. Este término suplanta la dicotomía entre transexual y travestido con un concepto de continuidad (...). Este sentimiento de intereses colectivos es importante para la comprensión del cambio histórico-cultural que se ha producido en las identidades de género y en la clarificación de la relación de la experiencia personal con la construcción social de la variancia de género (Bolin, 2003, p. 254).

El panorama identitario está lejos de ser unánime; las identidades se crean y se destruyen, se reflexionan y se transforman y, sin embargo, la agrupación de todas las personas trans se hace necesaria para la obtención de derechos de los que en la práctica están exentos.

Esta lucha y consecución de derechos por parte de minorías marginadas en el ámbito de la identidad de género pone sobre la mesa argumentos suficientes para criticar la sociología de la desviación; lo que antes era marginal, anormal, perverso, desviado y tantas calificaciones en línea empieza a estructurarse normativamente. Se ha pasado de rarezas y 
excentricidades subjetivas a comunidades reivindicativas.

\section{Notas}

${ }^{1}$ La filiación social no es algo novedoso. Las sociedades que estudiaron los antropólogos clásicos conocen y aplican estrategias para subvertir las imposiciones biológicas, como la esterilidad, creándose así lazos sobre bases no orgánicas (véase la interpretación de Bolin 2003- de los matrimonios entre hombres y entre mujeres descritos por Evans-Pritchard).

2 "A diferencia de la paternidad biológica...", dice Ritzer (2011: 209) en referencia a la consideración de Durkheim como "padre" de la sociología moderna, "...la de las disciplinas no es susceptible a las pruebas de ADN, por tanto, se considera una construcción social". Aunque uno se queda tentado de afirmar que el ADN es construcción social, consideraremos aquí que, incluso tratándose de una construcción social, la paternidad de la institucionalización del estudio de la sexualidad socio-cultural en la universidad española sería para el profesor Nieto. Incluso, utilizando un concepto de moda ("por defecto"), la paternidad habría que adjudicársela a él. A partir de ahí todas las propuestas son viables.

${ }^{3}$ En esos años una licenciatura de segundo ciclo era aquella estructurada en 2 cursos académicos $\left(4^{\circ}\right.$ y $\left.5^{\circ}\right)$ a la que se accedía con algunas diplomaturas (tres cursos académicos universitarios, $1^{\circ}, 2^{\circ}$ y $\left.3^{\circ}\right)$ específicas.

${ }^{4}$ En relación a estos datos, la Oficina de Tratamiento de la Información de la UNED, online y de acceso restringido, presenta unos datos de matrícula que exceden, con mucha diferencia, a los que aquí se presentan. Nuestros datos están obtenidos de las Actas de cada curso académico, documento en el que se recogen las matrículas confirmadas al final del período lectivo.

\section{Referencias}

Bazin, L., Mendes-Leite, R. y Quiminal, C. (2000). Déclinaisons anthropologiques des sexualités, Journal des anhropologues, 82-83, 924.

BOE (25 de noviembre de 2002). RESOLUCIÓN de 5 de noviembre de 2002, de la Universidad Nacional de Educación a Distancia, por la que se ordena la publicación del plan de estudios de Licenciado en Antropología Social y Cultural.

Bolin, A. (2003). La transversalidad de género. Contexto cultural y prácticas de género. En Nieto, J.A. (Ed.), Antropología de la sexualidad $y$ diversidad cultural (231-259). Madrid, España: Talasa. 
Collard, C. (2000). Kinship Studies au tournant de siècle. L'Homme, 154$155,635-658$.

Dayan-Herzbrun, S. (1991). La sexualité au regard des sciencies sociales. Sciences sociales y santé, 9(4), 7-22.

De la Calle (2011). Lecturas de "antropología de la sexualidad" como acontecimiento intelectual. Revista de investigación en educación, 2(9), 99-115.

Guasch, O. (1993). Para una Sociología de la Sexualidad. Revista Española de Investigaciones Sociológicas, 64, 105-121.

Guasch, O. y Viñuales, O. (2003). Introducción. Sociedad, sexualidad y teoría social: la sexualidad en perspectiva sociológica. En Sexualidades. Diversidad y control social (9-17). Barcelona, España: Bellaterra.

Herdt, G. (1999). Sexing Anthropology: Rethinking Sexual Culture, Subjectivity and the Method of Anthropological Participant Observation. En Suggs, D.N. y Miracle, A.W. (Eds.), Culture, Biology and Sexuality (17-32). USA: The University of Georgia Press.

Iglesias de Ussel, J. (1983). La sociología de la sexualidad en España: notas introductorias. Revista Española de Investigaciones Sociológicas, 21, 103-133.

Kottak, C.P. (2006). Antropología Cultural. Madrid, España: Mc Graw Hill.

Lévi-Strauss, C. (2012). La antropología ante los problemas del mundo moderno. Barcelona, España: RBA.

Mejía, N. (2006). Transgenerismos. Una experiencia transexual desde la perspectiva antropológica. Barcelona, España: Edicions Bellaterra.

Nieto, J.A. (2011). Sociodiversidad y sexualidad. Madrid, España: Talasa.

Nieto, J.A. (2004). Antropología de la Sexualidad y la Universidad española. En Anuario 2003. Centro de estudios superiores de México y Centroamérica (239-250). México: Universidad de Ciencias y Artes de Chiapas.

Nieto, J.A. (2003). Antropología de la Sexualidad y Diversidad Cultural. Madrid, España: Talasa.

Nieto, J.A. (2001). Homo proteico u homo prometeico? Reflexiones sobre Antropología de la Sexualidad desde una experiencia académica. En Cátedra, M. (Ed.), La mirada cruzada en la Península Ibérica (137158). Madrid, España: Los Libros de la Catarata. 
Nieto, J.A. (1998). Transexualidad, transgenerismo y cultura. Madrid, España: Talasa.

Nieto, J.A. (1997). Sex o no sex: el discurso de la sexualidad en Antropología. En Lameiras, M. y López, A. (Eds.). Sexualidad y Salud (35-47). Santiago de Compostela, España: Tórculo Edicions.

Nieto, J.A. y De la Calle, J. (2013). Transemociones/transentimientos. Estructura, acontecimiento y derechos humanos de las personas transgénero. Docta. Revista de Psicoanálisis, 9, 156-176.

Ritzer, G. (2011). Teoría Sociológica Clásica. Madrid, España: McGrawHill.

Touraine, A. (2005). Un nuevo paradigma para comprender el mundo de hoy. Barcelona, España: Paidós.

Vance, C. S. (1991). Anthropology Rediscovers Sexuality: a Theoretical Comment. Social Science and Medicine, 33(8), 857-884.

Weeks, J. (1993). El malestar de la sexualidad. Significados, mitos y sexualidades modernas. Madrid, España: Talasa.

Dr. Jaime de la Calle Valverde is Professor-Tutor in the associated centers of Cantabria, Portugalete and Vitoria, at the National University of Distance Education (UNED)

Contact Address: jdlcalle@poli.uned.es 